\title{
Identification of a novel pathogenic variant in PALB2 and $B A R D I$ genes by a multigene sequencing panel in triple negative breast cancer in Morocco
}

\author{
Abdelilah Laraqui ${ }^{1,2}$, Mathias Cavaillé3,4, Nancy Uhrhammer ${ }^{3,4}$, Oubaida ElBiad ${ }^{1,5}$, Yannick Bidet ${ }^{3,4}$, \\ Hicham El Rhaffouli2 ${ }^{2}$ Hicham El Anaz ${ }^{1}$, Driss Moussaoui Rahali ${ }^{6}$, Jaouad Kouach ${ }^{6}$, Khaled Guelzim ${ }^{6}$, \\ Bouabid Badaoui ${ }^{5}$, Abderrahman AlBouzidi7, Mohammed Oukabli ${ }^{7}$, Rachid Tanz ${ }^{8}$, Yasser Sbitti ${ }^{8}$, \\ Mohammed Ichou ${ }^{8}$, Khaled Ennibi ${ }^{1}$, Yassine Sekhsokh², Yves-Jean Bignon ${ }^{3,4}$
}

1. Unité de séquençage, Centre de virologie, des maladies infectieuses et tropicales, Hôpital militaire d'Instruction Mohammed V, Faculté de Médecine et de Pharmacie, Université Mohammed V, Rabat, Maroc.

2. Laboratoire de Recherche et de Biosécurité P3, Hôpital Militaire d'Instruction Mohammed V, Faculté de Médecine et de Pharmacie, Rabat, Maroc.

3. Laboratoire Diagnostic Génétique et Moléculaire, Centre Jean Perrin, 58 rue Montalembert, Clermont-Ferrand, France.

4. INSERM, U1240 Imagerie Moléculaire et Stratégies Théranostiques, Université Clermont Auvergne, Clermont-Ferrand, France.

5. Laboratoire de Biodiversité, Ecologie et Génome, Faculté des Sciences, Université Mohammed V, Rabat, Maroc.

6. Service de Gynécologie Obstétrique, Hôpital Militaire d'Instruction Mohammed V, Faculté de Médecine et de Pharmacie, Rabat, Maroc.

7. Laboratoire d'Anatomopathologie, Hôpital Militaire d'Instruction Mohammed V, Faculté de Médecine et de Pharmacie, Rabat 10000, Maroc.

8. Service d'Oncologie Médicale, Hôpital Militaire d'Instruction Mohammed V, Faculté de Médecine et de Pharmacie, Rabat, Maroc.

$\triangle$ Corresponding author: Abdelilah LARAQUI , Laboratoire de Recherche et de Biosécurité P3, Hôpital Militaire d'Instruction Mohammed V, Rabat, Maroc. Tel: (212) 678682 530; E-mail: loranjad@yahoo.fr.

(C) The author(s). This is an open access article distributed under the terms of the Creative Commons Attribution License (https://creativecommons.org/licenses/by/4.0/). See http://ivyspring.com/terms for full terms and conditions.

Received: 2021.04.29; Accepted: 2021.08.21; Published: 2021.09.18

\begin{abstract}
Pathogenic variants (PVs) in BRCA genes have been mainly associated with an increasing risk of triple negative breast cancer (TNBC). The contribution of PVs in non-BRCA genes to TNBC seems likely since the processing of homologous recombination repair of double-strand DNA breaks involves several genes. Here, we investigate the susceptibility of genetic variation of the BRCA and non-BRCA genes in 30 early-onset Moroccan women with TNBC.

Methods: Targeted capture-based next generation sequencing (NGS) method was performed with a multigene panel testing (MGPT) for variant screening. Panel sequencing was performed with genes involved in hereditary predisposition to cancer and candidate genes whose involvement remains unclear using Illumina MiSeq platform. Interpretation was conducted by following the American College of Medical Genetics and Genomics-Association for Molecular Pathology (ACMG-AMP) criteria.

Results: PVs were identified in $20 \%(6 / 30)$ of patients with TNBC. Of these, $16.7 \%(5 / 30)$ carried a BRCA PV [10\% (3/30) in BRCA I, 6.7\% (2/30) in BRCA2] and 6.6\% (2/30) carried a non-BRCA PV. The identified PVs in BRCA genes (BRCAl c.798 799delTT, BRCAl c.3279delC, BRCA2 c.1310 1313del, and BRCA2 c.1658T>G) have been reported before and were classified as pathogenic. The identified founder PVs BRCAl c.798_799del and BRCA2 c.1310_1313delAAGA represented 10\% (3/30). Our MGPT allowed identification of several sequence variations in most investigated genes, among which we found novel truncating variations in PALB2 and BARDI genes. The PALB2 c.3290dup and BARDI c.1333G >T variants are classified as pathogenic. We also identified 42 variants of unknown/uncertain significance (VUS) in 70\% (21/30) of patients with TNBC, including 50\% (21/42) missense variants. The highest VUS rate was observed in ATM (13\%, 4/30). Additionally, 35.7\% (15/42) variants initially well-known as benign, likely benign or conflicting interpretations of pathogenicity have been reclassified as VUS according to ACMG-AMP.
\end{abstract}

Conclusions: PALB2 and BARDI along with BRCA genetic screening could be helpful for a larger proportion of early-onset TNBC in Morocco.

Key words: Triple negative breast cancer, Next Generation Sequencing, Multigene panel testing, ACMG-AMP guidelines, Moroccan Population 


\section{Introduction}

Breast cancer (BC) is the most commonly diagnosed malignancy and the leading cause of cancer death among women worldwide. An estimated 19.3 million new cases and 10 million cancer deaths occurred in 2020 [1]. According to the GLOBOCAN Cancer Tomorrow prediction tool, incident cases are expected to increase by more than $46 \%$ by 2040 [2].The increasing global BC burden is mainly observed in low and medium human development index (HDI) countries [3], particularly women under the age of 50. Marked changes in lifestyle, socio-cultural contexts, and built environments are having a major impact on the prevalence of risk factors for $\mathrm{BC}$ burden in lowand medium HDI countries [4]. In North Africa (i.e., in Morocco, Algeria, Tunisia, Libya and Mauritania) $\mathrm{BC}$ has rapidly overtaken cervical cancer as the most commonly diagnosed cancer [5]. The incidence among women aged 15-49 is lower than in Western countries, but the very low incidence among women over 50, combined to the young age pyramid of North-Africa, makes the relative proportions of young patients substantially higher (50-60\% versus $20 \%$ in France) [5]. The size and grade of breast tumors are increased, while the median age of onset (48) is more than ten years younger than the European/North American median of 61 [6]. Moreover, the relative frequency of triple negative and inflammatory breast cancer is also higher in North Africa [5].

$\mathrm{BC}$ is a heterogeneous and polygenic disease that can be divided into different molecular sub-types based on histological and genomic features. Increasing steroid hormone receptors expression (estrogen and/or progesterone receptors) defines different BC subtypes using immunohistochemistry (IHC) markers together with clinic-pathologic indexes. Approximately $70 \%$ of $\mathrm{BCs}$ are estrogen receptors (ER) positive (ER+) and/or progesterone receptor (PR) positive (PR+) tumors [7]. The human epidermal growth factor receptor type (HER) 2 amplification defines a second type, with an incidence of about $20 \%$ of BCs [8]. The remaining belongs to the triple negative breast cancer (TNBC) subtype, i.e. those that do not over-express ER, PR or HER2. TNBC is associated with advanced-stage disease and higher-grade tumors at diagnosis and is associated with an increased recurrence risk and poor five-year survival rates relative to other BCs [9].

Current evidence suggests the association between specific molecular subtypes and $B R C A$ mutational status. BRCA1 PV carriers mainly increase TNBC, whereas BRCA2 carriers are more likely to increase ER+ and/or PR+ tumors [10]. Besides BRCA genes, an increasing number of studies have investigated genetic predisposition to TNBC using gene panel analysis $[11,12]$. PVs in non- $B R C A$ genes have been observed in women with TNBC, and subsequent studies showed that PVs in BARD1, BRIP1, PALB2, RAD51C, and RAD51D are more common in TNBC compared to other BC subtypes [13]. PVs in established BC genes as well as other cancer susceptibility genes were identified in $14.4 \%$ (8.4\% BRCA genes and $6.0 \%$ non-BRCA) of TNBC patients. PVs in BARD1, BRCA1, BRCA2, PALB2, and $R A D 51 D$ were associated with high risk (Odds Ratio (OR) > 5.0) of TNBC. PVs in BRIP1, RAD51C, and TP53 were associated with moderate risk $(\mathrm{OR}>2)$ of TNBC [11]. Moreover, PVs in BARD1, RAD51C, and $R A D 51 D$ placed the patient at moderate risk for ER-BC and TNBC. Conversely, PVs in ATM, CDH1, and CHEK2 were associated with ER+ BC. Additionally, there was a higher prevalence of PVs in BRCA1, BRCA/2 and PALB2 observed with TNBC compared with ER+ BC, $8.13 \%$ versus $1.84 \%$, respectively [14].

In North Africa, $B R C A$ mutations frequency varied widely from $\approx 1 \%$ (Morocco) in sporadic $B C$ [15] to $37.5 \%$ (Tunisia) in hereditary breast or/and ovarian cancer patients (HBOC) [16]. The pooled prevalence of $B R C A P V$ s among HBOC was $16 \%$ [17]. However, the contribution of $B R C A$ and non-BRCA genes to TNBC has not yet been determined. In a recent study from Morocco, it has been reported that $22 \%$ of TNBC patients harbor PVs in the BRCA genes using Ion AmpliSeq BRCA1 and BRCA2 Panel [18]. Thus, there is a great need to investigate the frequency and importance of PVs in BRCA and non-BRCA genes among TNBC patients. Herein, we present the first Next Generation Sequencing (NGS)-based Multigene Panel Test (MGPT) study of 30 early onset $(\leq 41$ years of age) Moroccan women with TNBC. Panel sequencing was performed with genes involved in hereditary predisposition to cancer and candidate genes whose involvement remains unclear. This provides an advantage to map susceptibility genes for TNBC in North Africa.

\section{Methods}

\section{Study subjects}

Newly diagnosed women with TNBC were identified at the department of Obstetrics and Gynecology of Mohammed V Military Teaching Hospital in Rabat. TNBC patients were chosen according to the following criteria: age at diagnosis $\leq$ 41 years without family history or belonging to a family history. This family history of cancer was defined as diagnosed BC or OC in first- and second-degree relatives. Clinico-pathological data of 
TNBC, including tumor site, histological type and grade and TNM classification were collected at BC diagnosis. A total of 30 early onset TNBC patients were enrolled between January 2020 and June 2020, including $18(60 \%)$ without a family history and 12 (40\%) with a family history. Informed consent was obtained from all participants at the time of peripheral blood draw. Patients consenting to participate in the study completed epidemiology and family history questionnaires and donated $10 \mathrm{ml}$ of blood for genetic analyses.

\section{Immunohistochemical analysis}

The status of ER, PR, and HER2 receptors was determined using IHC analysis. Briefly, IHC analysis to determine ER and PR status was performed using standard procedures on $4 \mu \mathrm{m}$ sections of paraffin embedded tissue specimens stained with the monoclonal antibodies 6F11 and 1A6 for ER and PR, respectively. Nuclear staining $1 \%$ was considered a positive result. The Hercept test was carried out in the institute since 2007 and was determined for all patients during the course of this study. Assays are scored with a 4 -tiered system $(0-3+)$. HER2 positivity was defined as strong complete membrane staining in at least $10 \%$ of tumor cells. Patients were considered HER2 if they had IHC 3+ by DAKO Hercept test. Tumors exhibiting equivocal HER2 expression, denoted as 2+ membranous staining of tumor cells, are confirmed by fluorescence in situ hybridization (FISH) at an outside laboratory. A signal ratios (HER2: CEP17) of $\geq 2.2$ were classified as amplified. In the absence of positive FISH data, tumors scored $2+$ by IHC were considered negative for HER2.

\section{DNA isolation}

Genomic DNA was isolated from $200 \mu \mathrm{l}$ peripheral blood anti-coagulated with Ethylene diamine tetra acetic acid on Blood DNA Maxi kit (Qiagen, Hilden, Germany) following the manufacturer's manual. DNA concentrations were assessed with the dsDNA HS assay kit by the Qubit 4.0 Fluorometer (Thermo Fisher Scientific, Waltham, USA).

\section{Gene panel testing}

We performed targeted capture sequencing with a gene panel that is associated with high, intermediate and low cancer risk and candidate genes whose involvement remains unclear. Gene panel was designed by "Département d'Oncogénétique, Centre Jean Perrin, Clermont-Ferrand, France" according to the literature.The mode of inheritance is dominant in AIP, APC, ATM, BAP1, BARD1,BMPR1A, BRCA1, BRCA2, BRIP1, CASR, CDC73, CDH1, CDK4,
CDKN2A, CHEK2, EPCAM, FANCM, FH, FLCN, MAX, MCIR, MEN1, MET, MITF, MLH1, MSH2, MSH6, MUTYH, NBN, NF1, NF2, PALB2, PMS2, POLD1, POLE, PTEN, RAD50, RAD51C, RAD51D, RET, SDHA, SDHAF2, SDHB, SDHC, SDHD, SMAD4, SMARCA4, STK11, TMEM127, TP53, and VHL. Additional genes such as BRK1, FAM175, GREM1, MLH3, MRE11, MMSH2, NTHL1, PMS1, RAD51, RAD51B, RINTI, RNF3, RNF43 and WRN were also included in the testing panel as candidate genes. The genes with a known association with $B C$ are $A T M$, BARD1, BRCA1, BRCA2, CHEK2, MSH6, NF1, PALB2, PTEN, RAD51C, RAD51D, and TP53. A list of analyzed genes is provided in Table 1.

\section{Panel sequencing}

Sonic fragmentation of DNA was performed on a Bioruptor instrument (Diagenode). Kapa HTP library preparation and SeqCap EZ Choice probes and reagents (Roche) were used for library preparation and capture. Briefly, 20ng of genomic DNA was fragmented and processed by end-repairing, A-tailing and adapter ligation of paired-end indexed adapters, and a 7-cycle pre-capture PCR amplification. Further, the libraries were enriched through hybrid capture based method using specific probes. This was followed by PCR based enrichment, cleanup, and quantification of double stranded DNA using high sensitivity Qubit (Invitrogen, USA) measurement. Agencourt AMPure XP beads (Beckman Coulter, USA) were widely used for PCR amplicon purification and DNA size selection. The quality of fragmentation, library, and capture were evaluated using the Agilent 2100 Bioanalyzer system. A final library concentration ranging from 8 to $10 \mathrm{pM}$ was used to carry out cluster generation and was sequenced on a Standard Flow Cell using V2 sequencing reagent kit (300 cycles) on MiSeq Instrument (Illumina, San Diego, CA). A typical sequencing run consists of 12 samples. All steps were performed following the providers' guidelines. No analysis of exons 11 to 15 of PMS2 and exons 1, 13, and 14 of SDHA, and no quantitative analysis of WRN exon 10 were performed, due to high identity with paralogs genes.

\section{Bioinformatics Analysis}

De-multiplexing was performed using bcl2fastq2 Conversion Software (Illumina). Sequencing reads were aligned to the University of California Santa Cruz hy19 reference genome assembly by BurrowsWheeler Aligner [19]. Recalibration of base quality scores (BaseRecalibrator) and realignment (RealignerTargetCreator, IndelRealigner) were carried out using Genome Analysis Toolkit (GATK) and PICARD tools, 
as recommended by Eurogentest guidelines [20]. A variant calling was made using GATK HaplotypeCaller and annotated using Ensemble Variant Effect
Predictor [21].Variants were filtered for quality score $\geq 30$, depth $\geq 30 x$, and presence in $\geq 20 \%$ of reads.

Table 1. Triple negative breast cancer susceptibility genes

\begin{tabular}{|c|c|c|c|c|}
\hline Gene & Genomic location & Coding transcript & Tumor types & Cancer syndrome \\
\hline AIP & $11 q 13.3$ & NM_003977.2 & Pituitary adenoma & pituitary syndrome \\
\hline$A P C$ & $5 q 22.2$ & NM_000038.5 & $\begin{array}{l}\text { colorectal, pancreatic, desmoid, } \\
\text { hepatoblastoma, glioma, other CNS }\end{array}$ & adenomatous polyposis coli; Turcot syndrome \\
\hline ATM & $11 \mathrm{q} 22.3$ & NM_000051.3 & $\begin{array}{l}\text { leukaemia, lymphoma, medulloblastoma, } \\
\text { glioma }\end{array}$ & ataxia-telangiectasia \\
\hline$B A P 1$ & $3 \mathrm{p} 21.1$ & NM_004656.2 & mesothelioma, uveal melanoma & tumor predisposition syndrome \\
\hline BARD1 & $2 q 35$ & NM_000465.2 & $\begin{array}{l}\text { ovarian cancer, breast cancer, endometrioid } \\
\text { cancer }\end{array}$ & ovarian cancer, breast cancer, endometrioid cancer \\
\hline BMPR1A & $10 \mathrm{q} 23.2$ & NM_004329.2 & gastrointestinal polyps & juvenile polyposis \\
\hline BRCA1 & $17 \mathrm{q} 21.31$ & NM_007294.3 & breast, ovarian & hereditary breast/ovarian cancer \\
\hline BRCA2 & $13 \mathrm{q} 13.1$ & NM_000059.3 & breast, ovarian, pancreatic, leukaemia & hereditary breast/ovarian cancer \\
\hline BRK1 & $3 p 25.3$ & NM_018462 & renal & Von Hippel-Lindau (VHL) syndrome \\
\hline BRIP1 & $17 q 23.2$ & NM_032043.2 & AML, leukaemia, breast & Fanconi anaemia J, breast cancer susceptiblity \\
\hline CASR & $3 q 21.1$ & NM_000388.3 & parathyroid, colorectal & hyperparathyroidism-jaw \\
\hline CDC73 & $1 \mathrm{q} 31.2$ & NM_024529.4 & $\begin{array}{l}\text { Parathyroid adenoma, multiple ossifying } \\
\text { jaw fibroma }\end{array}$ & hyperparathyroidism-jaw tumour syndrome \\
\hline $\mathrm{CDH} 1$ & $16 q 22.1$ & NM_004360.3 & gastric & familial gastric carcinoma \\
\hline CDK4 & $12 \mathrm{q} 14.1$ & NM_000075.3 & melanoma & familial malignant melanoma \\
\hline$C D K N 2 A$ & $9 \mathrm{p} 21.3$ & NM_000077.4 & melanoma, pancreatic & familial malignant melanoma \\
\hline CHEK2 & 22q12.1 & NM_007194.3 & breast & Familial breast cancer \\
\hline EPCAM & $2 \mathrm{p} 21$ & NM_002354.2 & colorectal & colorectal cancer, hereditary non polyposis, type 8 \\
\hline FANCM & $14 \mathrm{q} 21.3$ & NM_020937.2 & breast, ovarian & hereditary breast/ovarian cancer \\
\hline FAM175 & $4 q 21.23$ & & breast, ovarian & hereditary breast/ovarian cancer \\
\hline FH & $1 \mathrm{q} 43$ & NM_000143.3 & leiomyomatosis, renal & hereditary leiomyomatosis and renal cell cancer \\
\hline FLCN & $17 \mathrm{p} 11.2$ & NM_144997.5 & renal, fibrofolliculomas, trichodiscomas & Birt-Hogg-Dube syndrome \\
\hline GREM1 & $15 q 13.3$ & NM_013372 & colorectal & hereditary mixed polyposis syndrome \\
\hline MAX & $14 q 23.3$ & NM_002382.4 & pheochromocytoma & pheochromocytoma, susceptibility to \\
\hline$M C I R$ & $16 \mathrm{q} 24.3$ & NM_002386.3 & skin & familial malignant melanoma \\
\hline MEN1 & $11 \mathrm{q} 13.1$ & NM_000244.3 & $\begin{array}{l}\text { Thyroid adenoma, pituitary adenoma, } \\
\text { pancreatic islet cell, carcinoid }\end{array}$ & multiple endocrine neoplasia type 1 \\
\hline MET & $7 q 31$ & NM_001127500.1 & lung, gastric, colon & lung, gastric, and colon cancer \\
\hline MITF & $3 p 13$ & NM_000248.3 & melanoma & melanoma, cutaneous malignant, susceptibility to, 8 \\
\hline MLH1 & $3 \mathrm{p} 22.2$ & NM_000249.3 & $\begin{array}{l}\text { colorectal, endometrial, ovarian, central } \\
\text { nervous system }\end{array}$ & hereditary non-polyposis colorectal cancer, Turcot syndrome \\
\hline MLH3 & $14 q 24.3$ & NM_001040108.1 & colorectal, endometrial & $\begin{array}{l}\text { colorectal cancer, hereditary nonpolyposis, type } 7 \text {, } \\
\text { Endometrial Cancer }\end{array}$ \\
\hline MRE11 & $11 q 21$ & NM_005591.4 & colorectal & colorectal cancer \\
\hline MSH2 & $2 \mathrm{p} 21-\mathrm{p} 16$ & NM_000251.2 & colorectal, endometrial, ovarian & hereditary non-polyposis colorectal cancer \\
\hline MSH6 & $2 \mathrm{p} 16.3$ & NM_000179.2 & colorectal, endometrial, ovarian & hereditary non-polyposis colorectal cancer \\
\hline MUTYH & 1 p34.1 & NM_001128425.1 & Colorectal & adenomatous polyposis coli \\
\hline NTHL1 & $14 q 24.3$ & NM_001040108.1 & colorectal, breast & colorectal cancer, breast cancer, and colorectal polyposis. \\
\hline NBN & $8 \mathrm{q} 21.3$ & NM_002485.4 & $\begin{array}{l}\text { non-Hodgkin lymphoma, glioma, } \\
\text { medulloblastoma, rhabdomyosarcoma }\end{array}$ & nijmegen breakage syndrome \\
\hline NF1 & $17 \mathrm{q} 11.2$ & NM_000267.3 & neurofibroma, glioma & neurofibromatosis type 1 \\
\hline NF2 & $22 \mathrm{q} 12.2$ & NM_000268.3 & meningioma, acoustic neuroma & neurofibromin 2 (merlin) \\
\hline PALB2 & $16 \mathrm{p} 12.2$ & NM_024675.3 & $\begin{array}{l}\text { Wilms tumour, medulloblastoma, AML, } \\
\text { breast }\end{array}$ & Fanconi anaemia N, breast cancer susceptibility \\
\hline PMS1 & $2 q 31-q 33$ & NM_000534.4 & colorectal & hereditary nonpolyposis colorectal cancer type 3 \\
\hline PMS2 & $7 \mathrm{p} 22.1$ & NM_000535.5 & $\begin{array}{l}\text { Colorectal, endometrial, ovarian, } \\
\text { medulloblastoma, glioma }\end{array}$ & hereditary non-polyposis colorectal cancer, Turcot syndrome \\
\hline POLD1 & $19 q 13.33$ & NM_001256849.1 & colorectal & lynch syndrome \\
\hline POLE & $12 q 24.3$ & NM_006231.2 & colorectal & $\begin{array}{l}\text { colorectal cancer, adenomatous colorectal polyps, family } \\
\text { histories of colorectal cancer }\end{array}$ \\
\hline PTEN & $10 \mathrm{q} 23.31$ & NM_000314.4 & Harmartoma, glioma, prostate, endometrial & Cowden syndrome, Bannayan-Riley-Ruvalcaba syndrome \\
\hline RAD50 & $5 \mathrm{q} 31.1$ & NM_005732.3 & breast cancer & Nijmegen breakage syndrome-like disorder \\
\hline RAD51 & $15 q 15.1$ & NM_002875.5 & lung, colon, breast cancer & $\begin{array}{l}\text { lung adenocarcinoma, glioblastoma, colon adenocarcinoma, } \\
\text { breast invasive ductal }\end{array}$ \\
\hline RAD51B & $14 \mathrm{q} 23-\mathrm{q} 24.2$ & NM_002877.5 & lung, skin, bladder, endometrial, prostate & $\begin{array}{l}\text { lung adenocarcinoma, cutaneous melanoma, bladder } \\
\text { urothelial carcinoma, endometrial endometrioid } \\
\text { adenocarcinoma, prostate adenocarcinoma }\end{array}$ \\
\hline RAD51C & $17 q 22$ & NM_058216.1 & breast, ovarian cancer & breast-ovarian cancer, familial, susceptibility to, 3 \\
\hline RAD51D & $17 \mathrm{q} 12$ & NM_002878.3 & breast, ovarian cancer & breast-ovarian cancer, familial, susceptibility to, 4 \\
\hline RET & $10 \mathrm{q} 11.21$ & NM_020975.4 & medullary thyroid, papillary thyroid, & multiple endocrine neoplasia 2A/2B \\
\hline
\end{tabular}




\begin{tabular}{|c|c|c|c|c|}
\hline Gene & Genomic location & Coding transcript & Tumor types & Cancer syndrome \\
\hline & & & pheochromocytoma & \\
\hline RINT1 & $7 \mathrm{q} 22.3$ & NM_021930.6 & breast cancer, colon & breast cancer, Lynch syndrome \\
\hline RNF43 & $17 q 23.2$ & NM_017763.4 & colon, breast, pancreatic, endometrial, lung & $\begin{array}{l}\text { colon adenocarcinoma, breast cancer, pancreatic } \\
\text { adenocarcinoma, endometrial, lung adenocarcinoma }\end{array}$ \\
\hline SDHA & $5 p 15$ & NM_004168.2 & lung, colon, breast, pancreatic, bladder & $\begin{array}{l}\text { lung adenocarcinoma, colon adenocarcinoma, breast cancer, } \\
\text { pancreatic adenocarcinoma, bladder urothelial carcinoma }\end{array}$ \\
\hline SDHAF2 & $11 \mathrm{q} 12.2$ & NM_017841.2 & paraganglioma & familial paraganglioma \\
\hline$S D H B$ & $1 \mathrm{p} 36.13$ & NM_003000.2 & paraganglioma, pheochromocytoma & familial paraganglioma \\
\hline$S D H C$ & $1 \mathrm{q} 23.3$ & NM_003000.2 & paraganglioma, pheochromocytoma & familial paraganglioma \\
\hline$S D H D$ & $11 \mathrm{q} 23.1$ & NM_003002.2 & paraganglioma, pheochromocytoma & familial paraganglioma \\
\hline$S M A D 4$ & $18 \mathrm{q} 21.2$ & NM_005359.5 & gastrointestinal polyp & juvenile polyposis \\
\hline SMARCA4 & $19 \mathrm{p} 13.3$ & NM_001128844.1 & lung, colon, endometrial, bladder, breast & $\begin{array}{l}\text { lung adenocarcinoma, colon adenocarcinoma, endometrial } \\
\text { endometrioid adenocarcinoma, bladder urothelial } \\
\text { carcinoma, breast invasive ductal carcinoma }\end{array}$ \\
\hline STK11 & $19 \mathrm{p} 13.3$ & NM_000455.4 & $\begin{array}{l}\text { Jejunal hamartoma, ovarian, testicular, } \\
\text { pancreatic }\end{array}$ & Peutz-Jeghers syndrome \\
\hline TMEM127 & $2 \mathrm{q} 11.2$ & NM_017849.3 & pheochromocytoma, renal cell carcinoma & pheochromocytoma, susceptibility to \\
\hline TP53 & $17 \mathrm{p} 13.1$ & NM_000546.5 & $\begin{array}{l}\text { breast, sarcoma, adrenocortical carcinoma, } \\
\text { glioma, multiple other tumour types }\end{array}$ & Li-Fraumeni syndrome \\
\hline VHL & 3p25.3 & NM_000551.3 & renal, haemangioma, pheochromocytoma & Von Hippel-Lindau syndrome \\
\hline WRN & 8p12 & NM_000553.4 & colon, gastric & peritoneal cancer, colon cancer, and stomach cancer \\
\hline
\end{tabular}

\section{Interpretation of variants}

An Interpretation of variants was conducted by following the classification system recommended by the American College of Medical Genetics and Genomics-Association for Molecular Pathology (ACMG-AMP) Standards and Guideline for the Interpretation of Sequence Variants [22]. The process can result in 1 of 5 classifications: benign, likely benign, unknown/uncertain significance (VUS), likely pathogenic, and pathogenic. Likely benign and benign variants were not clinically reported. All classifications were ultimately evaluated by $\mathrm{AL}, \mathrm{MC}$ and referred to NU. The clinical significance of each sequence variant was also based on a set of criteria such as allele frequency as well as the information from clinical genome databases including ClinVar (https://www.ncbi.nlm.nih.gov/clinvar/).

\section{In silico prediction}

A potential clinical effect of VUS was evaluated by the analysis of the severity of the amino acid changes and their conservation across species. These analyses were performed using the Mutation Taster through Alamut ${ }^{\circledR}$ Visual V.2.11.0 including Alignment-Grantham variation Grantham deviation (Align GVGD; http://agvgd.iarc.fr/agvgd_input. php), Polymorphism Phenotyping-2 (Poly-Phen-2; http://genetics.bwh.harvard.edu/pph2/), and Sorting Intolerant from Tolerant (SIFT; http://blocks. fhcrc.org/sift/SIFT.html) scores. The Alamut interactive software provides results and/or links to the following databases used for variant annotation: Exome Aggregation Consortium (ExAC), Genome Aggregation Database (gnomAD: https://gnomad. broadinstitute.org/), Database of Short Genetic
Variation (dbSNP) and ClinVar.

\section{Complementary analysis}

Identified PVs were confirmed on a second patient sample. PVs in BRCAand BRCA2 geneswere examined by NGS with Ion AmpliSeq BRCA1 and BRCA2 Panel (Life Technologies). The Sanger sequencing was performed for the novel PV in PALB2 and BARD1 genes using a 3500xl instrument and Big Dye terminator kit 3.1 (Applied Biosystems).

\section{Statistical analysis}

The statistical analysis used the chi-squared test, with $p<0.05$ taken as the threshold for a significant difference.

\section{Results}

The mean age at diagnosis of TNBC was $38( \pm$ 2.8) years. Most TNBC patients were diagnosed with infiltrating ductal carcinoma (IDC). The Scarff-BloomRichardson grades II and III were predominant (33.3\% and $36.7 \%$ of cases, respectively). Axillary lymph nodes contained metastasis $(\mathrm{N}+)$ in $5(16.7 \%)$ cases. Unilateral BC was diagnosed in all patients, and one $(3.3 \%)$ had both BC and OC. The characteristics of the early onset TNBC patients are summarized in Table 2.

PVs were identified in $20 \%(6 / 30)$ of patients with TNBC. Of these, $16.7 \%(5 / 30)$ carried a BRCA PV [10\% $(3 / 30)$ in BRCA1, 6.7\% (2/30) in BRCA2] and $6.6 \%(2 / 30)$ carried anon-BRCA PV. The identified PVs in BRCA1 (BRCA1 c.798_799delTT, BRCA1 c.3279delC) and in BRCA2 (BRCA2 c.1310_1313del and BRCA 2 c. $1658 T>\mathrm{G}$ ) have been reported before and were classified as pathogenic (class 5). The identified founder BRCA1 c.798_799del and BRCA2 c.1310_1313delAAGA accounted for 10\% (3/30) of all 
identified PVs. The BRCA1 c.798_799del (p.Ser267LysfsX19) variant was found in two unrelated TNBC patients. Both carriers were diagnosed less than 38 years of age and showed a strong family history of BC. The BRCA1 c.798_799delTT variant, located in exon 11, is a frame-shift variant including two small deletions, two bases (TT) deletion. The deletion causes a frame-shift which changes a Serine to a Lysine at codon 267, and creates a premature stop codon at position 19 of the new reading frame. The BRCA1 c.798_799delTT variant, previously reported as BRCA1 917_918delTT using alternate nomenclature, has been reported in association with familial and early-onset $\mathrm{BC}$ and $\mathrm{OC}$ and has been described as a North African identified founder variant [15, 23-26].

Table 2. Demographic and clinical-pathologic characteristics of 30 TNBC samples

\begin{tabular}{ll}
\hline Characteristics & Patients \\
\hline Age & $38( \pm 2.8)$ \\
Family history & \\
Positive & $18(60 \%)$ \\
Negative & $12(40 \%)$ \\
Histologic & \\
IDC & $19(63.3 \%)$ \\
Lolular & $7(23.3 \%)$ \\
Medullar & $2(6.7 \%)$ \\
Others & $2(6.7 \%)$ \\
Tumour size & \\
T1 & $5(16.7 \%)$ \\
T2 & $10(33.3 \%)$ \\
T3 & $11(36.7 \%)$ \\
T4 & $4(13.3 \%)$ \\
SBR grading & \\
I & $7(23.3 \%)$ \\
II & $10(33.3 \%)$ \\
III & $11(36.7 \%)$ \\
IV & $2(6.7 \%)$ \\
Lymph node status & \\
Positive & $5(16.7 \%)$ \\
negative & $7(23.3 \%)$ \\
\hline
\end{tabular}

IDC: invasive ductal carcinoma; SBR: Scarff-Bloom-Richardson.

The BRCA1 c.3279delC (p.Tyr1094IlefsX15) variant was detected in a young woman diagnosed with TNBC at the age of 37 . The BRCA1 c.3279delC variant, located in exon 11 , is a frame-shift variant including one small deletion, one base (C) deletion. The deletion causes a frame-shift which changes a Tyrosine to an Isoleucine at codon 1094, and creates a premature stop codon at position 15 of the new reading frame. Using alternate nomenclature, The BRCA1 c.3279delC variant would be defined as BRCA1c.3390delC.

The BRCA2 c.1310_1313delAAGA (p.Lys437Ilefs) variant, a specific founder variant from the North-East of Morocco [27], was detected in early onset women diagnosed with both TNBC (at the age of 35) and OC (at the age of 38). The BRCA2 c.1310_1313delAAGA variant, located in exon 10, causes a frame-shift which changes a Lysine to an Isoleucine at codon 437 and creates apremature stop codon at position 22 of the new reading frame. It has previously reported as BRCA2 1310del4, BRCA21537del4, and BRCA21538del4 using alternate nomenclature.

The BRCA2 c.1658T>G (p.Leu553Ter) variant, located in exon 11, causes a non-sense substitution. It was observed in one patient without family history, diagnosed with TNBC at the age of 36. In databases, theBRCA2c.1658T $>\mathrm{G}$ variant was associated with breast-ovarian cancer, hereditary cancer-predisposing syndrome, or hereditary breast or/and ovarian cancer syndrome.

Our panel sequencing allowed identification of several sequence variations in most investigated genes, among which we found novel variation in PALB2 and BARD1 genes. The PALB2 c.3290dup (p.Lys1098) variant has not been reported before in the BIC database. The PALB2 c.3290dup variant was detected in a young patient diagnosed with TNBC at an age $\leq 36$ years. Family history was negative in the PV carrier. Thus, we concluded that this PV is not linked to family history of $\mathrm{BC}$ and/or OC. The PALB2 c.3290dup variant is a frame-shift variation due to the insertion of C nucleotide at acid 3290 of codon 1097 in exon 12, which is predicted to lead to a premature stop codon 1098 and a truncated protein. The BARD1 c.1333G > T (p.Glu445), that has not been reported previously, is classified as pathogenic (Class 5). The BARD1 c.1333G $>\mathrm{T}$ variant occurred with BRCA2 c.1658T $>\mathrm{G}$ (p.Leu553Ter) in early onset TNBC women diagnosed with pancreatic cancer at the age of 32 . The early disease onset and having two cancers in this case may be the result of harboring two PVs in BRCA2 and $B A R D 1$ genes. Details of PVs detected by NGS-based MGPT in our study are reported in Table 3.

We also identified 42 VUS in $70 \%(21 / 30)$ of TNBC patients, including $50 \%(21 / 42)$ missense variants. The other VUSs were characterized as intronic or synonymous variants. A high rate was expected given the number of genes included in our panel gene. The BC susceptibility gene carrying the highest number of VUS was ATM $(13 \%, 4 / 30)$. Additionally, 35.7\% (15/42) variants initially wellknown as benign, likely benign or conflicting interpretations of pathogenicity have been reclassified as VUS according to the ACMG-AMP classification. Details of the identified VUS are shown in Table 4. 
Table 3. Pathogenic variants and likely pathogenic variant detected by NGS based-MGPT in Moroccan TNBC patients

\begin{tabular}{|c|c|c|c|c|c|c|c|c|}
\hline & $\begin{array}{l}\text { Age at } \\
\text { diagnosis }\end{array}$ & $\begin{array}{l}\text { Affected } \\
\text { gene }\end{array}$ & Nucleotide change & $\begin{array}{l}\text { Amino acid } \\
\text { change }\end{array}$ & Type of variant & Rs number & ClinVar Database & $\begin{array}{l}\text { ACMG } \\
\text { Classification }\end{array}$ \\
\hline 1907L0132 & 38 & BRCA1 & c.798_799del & p.Ser267fs & Frameshift & rs80357724 & Pathogenic & 5 \\
\hline 1907L0131 & 36 & BRCA1 & c.798_799del & p.Ser267fs & Frameshift & rs80357724 & Pathogenic & 5 \\
\hline 1907L0133 & 40 & BRCA1 & c.3279del & p.Tyr1094Ilefs & Frameshift & rs397509050 & Pathogenic & 5 \\
\hline 1907L0141 & 38 & BRCA2 & c.1310_1313del & p.Lys437Ilefs & Frameshift & rs80359277 & Pathogenic & 5 \\
\hline \multirow[t]{2}{*}{ 1907L0145 } & 37 & BRCA2 & c. $1658 \mathrm{~T}>\mathrm{G}$ & p.Leu553Ter & Nonsens & rs876659627 & Pathogenic & 5 \\
\hline & & BARD1 & c. $1333 \mathrm{G}>\mathrm{T}$ & p.Glu445 & Missense & Not reported & Not reported & 5 \\
\hline 1907L0146 & 40 & PALB2 & c.3290dup & p.Lys1098 & Frameshift & Not reported & Not reported & 5 \\
\hline
\end{tabular}

ACMG: American College of Medical Genetics and Genomics.

Table 4. Variant of unknown/uncertain significance detected by NGS based-MGPT in Moroccan TNBC patients

\begin{tabular}{|c|c|c|c|c|c|c|}
\hline Gene & Sequence variant & Amino acid hange & Type of variant & Rs number & ClinVar Database & $\begin{array}{l}\text { ACMG } \\
\text { Classification }\end{array}$ \\
\hline$A P C$ & c. $-151 G>C$ & Non coding & 5 Prime UTR & rs1029997545 & Likely benign & 3 \\
\hline$A P C$ & c. $325 \mathrm{C}>\mathrm{T}$ & p.Arg109Trp & Missense & Not reported & Not reported & 3 \\
\hline$A P C$ & c. $295 \mathrm{C}>\mathrm{T}$ & p.Arg99Trp & Missense & rs139196838 & Conflicting interpretations of pathogenicity & 3 \\
\hline$A P C$ & c. $835-41 \mathrm{~A}>\mathrm{G}$ & Non coding & Intron Variant & Not reported & Not reported & 3 \\
\hline$A P C$ & c. $781-41 \mathrm{~A}>\mathrm{G}$ & Non coding & Intron Variant & Not reported & Not reported & 3 \\
\hline ATM & c. $1595 \mathrm{G}>\mathrm{A}$ & p.Cys532Tyr & Missense & rs35963548 & Conflicting interpretations of pathogenicity & 3 \\
\hline ATM & c. $1810 \mathrm{C}>\mathrm{T}$ & p.Pro604Ser & Missense & rs2227922 & Conflicting interpretations of pathogenicity & 3 \\
\hline ATM & c. $9002 \mathrm{G}>\mathrm{A}$ & p.Ser3001Asn & Missense & rs587781413 & VUS & 3 \\
\hline ATM & c. $8560 \mathrm{C}>\mathrm{T}$ & p.Arg2854Cys & Missense & rs201958469 & VUS & 3 \\
\hline BARD1 & c. $1028 \mathrm{C}>\mathrm{T}$ & p.Thr343Ile & Missense & rs201032007 & Conflicting interpretations of pathogenicity & 3 \\
\hline$B R C A 1$ & c. $3587 \mathrm{C}>\mathrm{T}$ & p.Thr1196Ile & Missense & rs80356944 & VUS & 3 \\
\hline BRIP1 & c. $415 \mathrm{~T}>\mathrm{G}$ & p.Ser139Ala & Missense & rs202072866 & VUS & 3 \\
\hline$B M P R 1 A$ & c. $431-30 A>G$ & Non coding & Intron Variant & Not reported & VUS & 3 \\
\hline CDKN2A & c. $13 \mathrm{~T}>\mathrm{A}$ & p.Phe5Ile & Intron Variant & rs776987532 & VUS & 3 \\
\hline$C D K N 2 A$ & c.369T $>$ A & p.His123Gln & Missense & rs6413463 & Conflicting interpretations of pathogenicity & 3 \\
\hline FANCM & c. $1667 A>G$ & p.Asp556Gly & Missense & rs148810507 & VUS & 3 \\
\hline FANCM & c. $1576 C>G$ & p.Leu526Val & Missense & rs144215747 & VUS & 3 \\
\hline MET & c. $841 \mathrm{~T}>\mathrm{G}$ & p.Phe281Val & Missense & Not reported & Not reported & 3 \\
\hline MITF & c. $-28 \mathrm{C}>\mathrm{T}$ & Non coding & 5 Prime UTR & Not reported & Not reported & 3 \\
\hline MLH3 & c. $3746 \mathrm{C}>\mathrm{T}$ & p.Ser1249Phe & Missense & rs139265757 & Benign & 3 \\
\hline MSH2 & c. $965 \mathrm{G}>\mathrm{T}$ & p.Gly322Val & Missense & rs4987188 & VUS & 3 \\
\hline MSH6 & c. $2540 \mathrm{~A}>\mathrm{T}$ & p.Glu847Val & Missense & Not reported & Not reported & 3 \\
\hline$N B N$ & c. $425 \mathrm{~A}>\mathrm{G}$ & p.Asn142Ser & Missense & rs769414 & Conflicting interpretations of pathogenicity & 3 \\
\hline NF1 & c. $8161-45 \mathrm{~A}>\mathrm{C}$ & Non coding & Intron Variant & rs17879551 & Likely benign & 3 \\
\hline NF1 & c. $8098-45 \mathrm{~A}>\mathrm{C}$ & Non coding & Intron Variant & rs17879551 & Likely benign & 3 \\
\hline NTHL1 & c. $86 \mathrm{C}>\mathrm{T}$ & p.Pro29Leu & Missense & Not reported & Not reported & 3 \\
\hline PMSI & c. $-99 \mathrm{G}>\mathrm{T}$ & Non coding & 5 prime UTR & rs577363454 & VUS & 3 \\
\hline PMS2 & c. $250 \mathrm{~A}>\mathrm{C}$ & p.Thr84Pro & Missense & rs1554304938 & VUS & 3 \\
\hline POLD1 & c. $883 \mathrm{G}>\mathrm{A}$ & p.Val295Met & Missense & rs199545019 & Conflicting interpretations of pathogenicity & 3 \\
\hline POLD1 & c. $2388+5 G>A$ & Non coding & Intron Variant & rs750085275 & VUS & 3 \\
\hline POLD1 & c. $1014 C>G$ & p.Cys338Trp & Missense & Not reported & Not reported & 3 \\
\hline RAD50 & c. $2354 \mathrm{C}>\mathrm{T}$ & p.Ala785Val & Missense & Not reported & Not reported & 3 \\
\hline RAD51B & c. $1050 \mathrm{C}>\mathrm{G}$ & p.Cys350Trp & Missense & Not reported & Not reported & 3 \\
\hline RET & c. $1756 \mathrm{C}>\mathrm{T}$ & Leu586Phe & Missense & rs777604634 & VUS & 3 \\
\hline RNF43 & c. $172 A>G$ & Thr58Ala & Missense & Not reported & VUS & 3 \\
\hline RNF43 & c. $2054 \mathrm{C}>\mathrm{A}$ & Thr685Asn & Missense & Not reported & Not reported & 3 \\
\hline RNF43 & c. $-611 C>T$ & Non coding & Missense & rs62636625 & Not reported & 3 \\
\hline SDHA & c. $1367 \mathrm{C}>\mathrm{T}$ & Ser456Leu & Missense & rs76896145 & Benign & 3 \\
\hline SMARCA4 & c.722_733del & Gly241_Pro244del & Inframe Deletion & rs568390760 & Conflicting interpretations of pathogenicity & 3 \\
\hline SMARCA4 & c. $-72 \mathrm{C}>\mathrm{T}$ & Non coding & 5 prime UTR & rs559144002 & Likely benign & 3 \\
\hline$W R N$ & c.1530_1532del & Glu510del & Initiator Codon & rs781777438 & VUS & 3 \\
\hline WRN & c. $3785 \mathrm{C}>\mathrm{G}$ & Thr1262Arg & Missense & rs78488552 & Conflicting interpretations of pathogenicity & 3 \\
\hline
\end{tabular}

ACMG: American College of Medical Genetics and Genomics; VUS: variant of uncertain significance.

\section{Discussion}

The absence of specific molecular markers for TNBC has made the targeted treatments extremely challenging and the death rates very high compared to the other BC subtypes. The NGS offers several clinical applications in cancer and precision oncology that are significant for risk predictors, early detection of disease, diagnosis by sequencing and medical imaging, accurate prognosis, biomarker identification and identification of therapeutic targets for novel drug discovery [28]. To identify TNBC patients who 
might benefit from treatment strategies, $B R C A$ and non-BRCA genes testing through NGS could lead to a more accurate prediction of the responsiveness to platinum and poly (ADP-ribose) polymerase (PARP) inhibitors. Thus, this strategy should be considered in management and precision medicine [29]. Recent data have hypothesized that patients with advanced-stage TNBC associated with PVs in BRCA genes might be specifically sensitive to PARP inhibition; both Olaparib and Talazoparib are currently approved for such situation [30,31]. Moreover, there is some evidence that adding platinum-agents in the neoadjuvant setting improves the pathologic complete response [32,33]. The role of PARP inhibitors in the setting of non- $B R C A$ associated cancers has been limited. Recently, Lapatinib plus Veliparib therapy have a manageable safety profile and promising antitumor activity in advanced TNBC [34].

\section{TNBC and BRCA genes}

Genetic susceptibility to TNBC has been associated with rare germline variants occurring in $B R C A$ genes, and BRCA2 PVs were less common than BRCA1 PVs [10,35]. In our study, the BRCA PV prevalence was $16.7 \%$ among TNBC women aged $<41$. Among $B R C A$ carriers, $B R C A 1$ gene was found to be mutated in $10 \%(3 / 30)$, while BRCA2 gene was mutated in $6.7 \%(2 / 30)$. Our findings highlight that the TNBC phenotype at young age at onset can provide a valuable tool for identifying individuals with high likelihood of being BRCAPV carriers. This information emphasizes the recommendation for genetic testing in women diagnosed with TNBC at a young age because they have an increased risk of carrying $B R C A$ PVs particularly in BRCA1. Data from a previous Tunisian study showed that diagnosis before the age of 40 could be the effective $B R C A$ testing selection criterion among women with triple-negative tumors [36]. Recent findings highlight that receptor triple negative could be an effective selection of patients for BRCA1 analysis and should therefore be considered in genetic screening guidelines in Tunisia [37]. In Algeria, the BRCA1 PVs have been detected in Algerian patients with TNBC diagnosed at age $\leq 45$ [38]. Thus, an earlier age should be considered as a guideline for $B R C A$ genetic testing in women with TNBC in North Africa.

By analyzing the incidence rates of BRCA1 PVs in both TNBC and non-TNBC, Tun et al. found that women with high-risk TNBC are much more likely to have PVs in BRCA1 gene compared with women with non-TNBC and provides a relative risk of 5.65 (95\% $\mathrm{CI}, 4.15-7.69)$. Furthermore, two out of nine $(\approx 22 \%)$ TNBC patients harbor a PV in BRCA1 gene [39].
Armstrong et al. reported a concordant finding, although the estimates of BRCA1 PV prevalence were mostly lower than the estimate by Tun et al. [40]. In four studies of TNBC patients that reported on BRCA PVs prevalence, values ranged from $9.3 \%$ in an Australian study $(n=439)$ [35] to $15.4 \%$ in a US study $(n=207)$ [41]. Both of these studies were done in early and advanced tumor stages BC populations. In UK, Robertson et al. showed that diagnosis of TNBC below 50 years would be a suitable age threshold for $B R C A$ testing and may be a cost effectiveness strategy [42]. Lu et al. recommended genetic testing for TNBC patients diagnosed before the age of 50 , a population with $B R C A$ PV frequency of $17.5 \%$. The authors also highlighted data showing that the estimated BRCA PV frequency of individuals with any type of $B C$ diagnosed before the age of 40 is $11 \%$ [43]. Overall, testing in the UK has an unusually high threshold in comparison with the other European countries, where in all cases $<41$ years would be eligible for screening [44].

When stratified by family history, our study showed that $13 \%(4 / 30)$ early onset TNBC patients with a family history were identified with PVs in $B R C A$ genes, compared to $3.3 \%(1 / 30)$ without a family history. Couch et al. revealed that $12.2 \%$ $(66 / 539)$ TNBC patients with a family history carry BRCA PVs, compared to $8.6 \%$ (83/969) patients without a family history [12]. Hartman et al. identified 21 BRCA PVs (13 in BRCA1 and 8 in BRCA2) in a cohort of 199 unselected women with TNBC and providing an overall prevalence rate of $10.6 \%$. Additionally, 5.2\% (8/153) PVs were found in $153 / 199$ patients without significant family history [45]. In an unselected cohort study in 77 TNBC patients, it was found that $19.5 \%(15 / 77)$ had $B R C A$ PVs including $15.6 \%(12 / 77)$ in BRCA1 and $3.9 \%$ $(3 / 77)$ in BRCA [46]. In a Canadian TNBC cohort $(\mathrm{n}=54)$ with no familial BC aggregation, 9\% (5/54) with PVs in BRCA1 and 2\% (1/54) of patients with PVS in BRCA2 were detected [47]. Lu et al. stated the importance of $B R C A$ testing regardless of family history as few female family members or small families can mask genetics relative to BC and OC [43]. Muendlein et al. conclude that the prevalence of $B R C A$ PVs is high in TNBC patients and are not restricted to young women or patients with a positive family history [48]. Although family history is commonly used to identify individuals with a possible predisposition to $\mathrm{BC}$, Zang et al. showed that family history could not predict an underlying predisposition cancer syndrome in most patients. Furthermore, some individuals with cancer have de novo mutations, whereas others inherit them with incomplete penetrance; where, the family history is 
likely to be negative [49].

\section{TNBC and non BRCA-genes}

In recent years it has become clear that truncating PALB2 variants have been shown to be associated with a high risk for breast, ovarian and pancreatic cancers [50-52]. In our investigation, the truncating variant PALB2 c.3290dup was found in $3.3 \%(1 / 30)$ of TNBC cases. According to the ACMGAMP guidelines, the PALB2 c.3290dup variant was characterized as pathogenic (class 5) and considered predisposing for TNBC. Family history was negative in a PALB2 c.3290dup carrier. Genetic testing for $P A L B 2$ would provide another key genetic marker to identify women at elevated risk of TNBC regardless of their family history of $\mathrm{BC}$. Our results suggest that the PALB2 gene contributes to the risk of TNBC in North Africans and highlights the need to identify PVs in the PALB2gene which has a significant impact on an individual's risk of TNBC in younger women.

Similar observations are made when comparing the frequency of PVs reported here to that reported in the study of Zanati et al. in which $4.3 \%$ of TNBC patients carried PVs in PALB2 [52]. The observed frequency of PALB2 PVs in our study $(3.3 \%)$ and the last one appears to be higher compared to other populations $(1.3-1.4 \%)$ [11,13]. Low prevalence of PALB2 germline mutation ( 1\%) was observed in 347 Australian TNBC women, similar to the prevalence of $P A L B 2$ germline mutation of $1 \%$ in familial non- $B R C A$ genes $\mathrm{BC}$ cohorts [11]. PALB2 PVs might be overrepresented in patients with TNBC in earlier studies performed in European cohorts [54,55]. It is estimated that $30 \%$ [56] and 34\% [57] of BC patients with a germline PALB2 PV have a TNBC subtype. Shimelis et al. found that PVs in PALB2 were found to be associated with a high-risk of TNBC with an OR of 14.41 (95\%CI; 9.27-22.60) and were enriched in patients with TNBC compared to non-TNBC tumors with an OR of $2.12(95 \% \mathrm{CI} ; 1.63-2.74)$ [11]. The relatively poor survival from $B C$ in patients with a PALB2 PV was not attributable to the high prevalence of triple-negative phenotype [57]. Due to the lower PV carrier frequency in the PALB2 gene in the population, broad-based studies are needed to refine the genetic testing criteria and the management of the patients and their family members.

$B A R D 1$ targeted sequencing studies showed that PVs in BARD1 gene are enriched in TNBCs, which are associated with higher rates of recurrence, progression, and mortality $[12,13]$. BARD1 PVs were increased by more than threefold in TNBC cases $(0.67 \%)$ compared to non-TNBC cases $(0.18 \%)$, suggesting that BARD1 is a predominantly TNBC predisposition gene [11]. In our study, the
BARD1c.1333G $>\mathrm{T}$ variant was identified in 3.3\% $(1 / 30)$ of TNBC women. According to the ACMG-AMP guidelines, BARD1 c.1333G $>\mathrm{T}$ was characterized as pathogenic (class 5).

Similar observations are made when comparing thefrequency of $B R A D 1 \mathrm{PVs}$ reported here to that reported in previous studies. De Brakeleer et al. suggest that TNBC patients are enriched for PVs in BRAD1 ascompared to control samples and high BCrisk families when they identified four harbored variants in $B A R D 1$, of which two protein-truncating variants (c.1347A $>\mathrm{G}$ and c.1972C $>\mathrm{T}$ ) have been confirmed as pathogenic [58]. In a study of 105 women with TNBC from a trial exploring the antitumor activity of neoadjuvant carboplatin/docetaxel chemotherapy, BARD1 PVs were detected in two TNBC patients (1.9\%) [59]. The Analysis of 1824 TNBC patients unselected for age or family history of $\mathrm{BC}$ led to the identification of $0.5 \%(9 / 1824)$ cases with a BARD1-truncating variant [12]. In Buys's study, the prevalence of BARD1 PVs was higher among women with TNBC (3.3\%) than among women with non-TNBC (1.7\%) [13]. Shimelis et al. identified 25 individuals harboring BARD1 PVs $(0.61 \%)$ and reported an OR of 5.92 for TNBC cases of African American and Caucasian populations [11]. Rofes et al. identified ten BARD1 PV carriers from 680 TNBC patients (carrier frequency $=0.9 \%$ ), resulting in an OR $=5.40$ [60]. Similar observations were reported in other studies [61,62], indicating that $B A R D 1$ is a risk gene for TNBC. Although the BARD1gene offers a new hope for improving the TNBC therapy, the low number of BARD1 PV carriers, the non-identification of a recurrent hotspot variant, a study with an sufficient sample size, a lack of geographically matched population controls have complicated the interpretation of the results and could hamper the strength of the association of BARD1 PVs with TNBC risks [56]. Further studies in larger cohorts will be necessary to more precisely assess the BARD1associated risk with this tumor phenotype.

\section{TNBC and VUS}

VUS represent a significant proportion of variants identified in clinical genetic testing, which account for about $40 \%$ of the total variants [63]. In our study, 42 VUS were identified in $70 \%(21 / 30)$ of TNBC patients including $50 \%(21 / 42)$ distinct missense variants. The gene with the highest VUS frequency was the $\operatorname{ATM}(13 \%, 4 / 30)$. The ATM is a very large gene and is one of the genes with more identified VUS (40\%) including missense, in-frame, or synonymous mutations [63]. In our MGPT, the genes that contain more VUS are among the top ten genes cited in the literature with the highest number of 
variants submitted. A previous study has shown that the rate of VUSs was proportional to the number of genes analyzed in the MGPT in TNBC [64]. Identification of the VUS has become a daily fact of life when tailoring genetic counseling, but little guidance is available for how best to approach them, and limited data are available on how they are affecting the medical practice and the well-being of cancer patients [65]. In the lack of reliable clinical information or functional evidence the VUS remains non-informative in risk management and decision making.

Management recommendations for diagnostic and treatment decision-making for the carriers of PVs in BRCA and non BRCA-genes have been established. Recommendations are focused on a combination of annual magnetic resonance imaging (MRI) and mammography for women with familial risk or $B R C A$ PV and a history of BC [66-68]. Guidelines also made recommendations for the treatment of individuals with a $B R C A$ carrier or those with a strong familial risk of developing $B C$. More recent European and US NCCN guidelines have updated recommendations regarding BRCA-targeted PARP inhibitor therapy in $B C$ [69]. Current evidence suggests that the $B C$ risk for PALB2 mutation carriers may overlap with that for $B R C A 2$ mutation carriers, particularly in the context of a significant family history [55]. Accordingly, recently introduced NCCN practice guidelines suggest that a PALB2 carrier should undergo a MRI or a mammography screening $[70,71]$. Several studies have shown that the BARD1 can potentially become a new target for $\mathrm{BC}$ treatment. Li et al. have reported that the BARD1 BRCT domain interacts with PAR, which results in a subsequent recruitment of the $B A R D 1-B R C A 1$ complex to the damaged DNA [72]. A higher $B A R D 1$ and $B R C A 1$ expression is associated with a worse prognosis of early $B C$ patients, especially the ones that received a radiotherapy, indicating the potential use of PI3K inhibitors to reverse chemoresistance and radioresistance in $\mathrm{ER}^{+} \mathrm{BC}$ patients [73]. Mammography and MRI remain the fundamental imaging modalities for the high andvery high-risk BC patients. An interesting approach might be radiogenomics, which brings together the clinical assessment, imaging results, and the genetic background [74]. This approach would be of interest in relation to the immunohistochemical staining of the $B A R D 1$ gene, which in turn can be imaged inmagnetic resonance scans [75].

\section{Limitations}

Our study has some limitations that should be acknowledged. The sample size was relatively small. Thus, our data underscore the need for larger series to better understand the frequency and the contribution of PVs in BRCA, PALB2 and BARD1 genes in patients with early onset TNBC. Despite the reduced sample size in our cohort, our findings supports those deleterious PVs in PALB2 and BARD1were enriched in TNBC patients. Our analysis does not include copy number variants. Although having many advantages, large sequencing panels still have limitations compared to the traditional Sanger sequencing test or smaller NGS panels in cancer precision. In our MGPT, Some genes or exons are not well captured and therefore are not covered, resulting in some variants within these regions going undetected and hence being refractory to analysis. Despite these limitations, our findings may help in implementing effective strategies for BRCA, PALB2 and BARD1 PVs testing in TNBC patients.

\section{Conclusion}

Defects in homologous recombination DNA repair genes that may be targeted with PARP inhibitors occur in 60 to $69 \%$ of TNBC. PARP repairs the damaged DNA and renders the tumor highly sensitive to platinum-based chemotherapy. With the rise of NGS, it is possible to target multiple genes rapidly and simultaneously in a cost-effective manner. Defining groups of TNBC patients with $B R C A, P A L B 2$ and BARD1 PVs is important for the clinical management of patients because several new treatment strategies are being evaluated for related tumors. Overall, an improvement in the detection rate by using an extensive panel analysis determines the hereditary cancer to which the patients and families are exposed based on their history and genetics as part of a targeted therapy [76].

\section{Supplementary Material}

Supplementary figure $\mathrm{s} 1$.

http://www.jgenomics.com/v09p0043s1.pdf

Supplementary table S2.

http://www.jgenomics.com/v09p0043s2.xlsx

Supplementary table S3.

http://www.jgenomics.com/v09p0043s3.xlsx

\section{Acknowledgements}

We thank the patients and their families for their participation. We also thank Marie Noelle ALLIRAND and Edwig FRETT of the Laboratoire Diagnostic Génétique et Moléculaire, Centre Jean Perrin, Clermont-Ferrand, France, for their support.

\section{Funding}

This research has been supported by Laboratoire de Recherche et de Biosécurité, Hôpital Militaire d'Instruction Mohammed V, Rabat, Maroc and 
Département d'Oncogénétique, Centre Jean Perrin, Clermont-Ferrand, France.

\section{Author contributions}

$\mathrm{AL}, \mathrm{NU}, \mathrm{OE}, \mathrm{YS}$ and Y-JB designed the study. $\mathrm{AL}, \mathrm{NU}, \mathrm{MC}$, and $\mathrm{OE}$ wrote this paper. JK, DMR, KG, MI, RT, YS, MO, and AA provided samples. AL, MC, $\mathrm{NU}, \mathrm{YB}$, and $\mathrm{RH}$ analyzed the data. $\mathrm{RH}$ and $\mathrm{HA}$ reviewed the manuscript. All authors read and approved the manuscript.

\section{Competing Interests}

The authors have declared that no competing interest exists.

\section{References}

1. Bray F, Ferlay J, Soerjomataram I, Siegel RL, Torre LA, Jemal A, Global cancer statistics 2018: GLOBOCAN estimates of incidence and mortality worldwide for 36 cancers in 185 countries.CA Cancer J Clin. 2018; 68: 394-424.

2. Heer E, Harper A, Escandor $N$, Sung H, McCormack V, FidlerBenaoudia MM. Global burden and trends in premenopausal and postmenopausal breast cancer: a population-based study. Lancet GlobHealth. 2020;8:e1027-e1037.

3. Li N, Deng Y, Zhou L, Tian T, Yang S, Wu Y, et al. Global burden of breast cancer and attributable risk factors in 195 countries and territories, from 1990 to 2017: results from the Global Burden of Disease Study 2017. J HematolOncol.2019;12:140.

4. [internet] https://www.iarc.who.int/wp-content/uploads/2020/12/pr292_ E.pdf.

5. Corbex M, Bouzbid S, Boffetta P. Features of breast cancer in developing countries, examples from North-Africa.EurJ Cancer. 2014;50:1808-18.

6. [Internet] Caducee. http://www.caducee.net/

7. Johnston SR, Dowsett M. Aromatase inhibitors for breast cancer: lessons from the laboratory. Nat Rev Cancer. 2003;3:821-31.

8. Goldhirsch A, Winer EP, Coates AS, Gelber RD, Piccart-Gebhart M, Thürlimann B, et al. Personalizing the treatment of women with early breast cancer: highlights of the St Gallen International Expert Consensus on the Primary Therapy of Early Breast Cancer 2013. Ann Oncol. 2013;24:2206-23.

9. Li X, Yang J, Peng L, Sahin AA, Huo L, Ward KC. Triple-negative breast cancer has worse overall survival and cause-specific survival than non-triple-negative breast cancer. Breast Cancer Res Treat. 2017;161:279-287.

10. Mavaddat N, Barrowdale D, Andrulis IL, Domchek SM, Eccles D, Nevanlinna $\mathrm{H}$, Ramus SJ, et al. Pathology of breast and ovarian cancers among BRCA1 and BRCA2 mutation carriers: results from the Consortium of Investigators of Modifiers of BRCA1/2 (CIMBA). Cancer Epidemiol Biomarkers Prev. 2012;21:134-47.

11. Shimelis, H,LaDuca $\mathrm{H}, \mathrm{Hu}, \mathrm{C}$, Hart S.N, Na J, Thomas, A, et al. Triple-Negative Breast Cancer Risk Genes Identified by Multigene Hereditary Cancer Panel Testing. J Natl Cancer Inst. 2018;110:855-862.

12. Couch FJ, Hart SN, Sharma P, Toland AE, Wang X, Miron P, et al. Inherited mutations in 17 breast cancer susceptibility genes among a large triple negative breast cancer cohort unselected for family history of breast cancer. J ClinOncol. 2015;33:304-311.

13. Buys SS, Sandbach JF, Gammon A, Patel G, Kidd J, Brown KL, et al. A study of over 35,000 women with breast cancer tested with a 25-gene panel of hereditary cancer genes. Cancer. 2017;123:1721-30.

14. Lee A, Moon BI, Kim TH. BRCA1/BRCA2 Pathogenic Variant Breast Cancer: Treatment and Prevention Strategies. Ann Lab Med. 2020;40:114-121.

15. Laraqui A, Uhrhammer N, Lahlou-Amine I, EL Rhaffouli H, El Baghdadi J, Dehayni M, et al. Mutation screening of the BRCA1 gene in early onset and familial breast/ovarian cancer in Moroccan population. Int $\mathrm{J}$ Med Sci. 2013;10:60-67.

16. Troudi W, Uhrhammer N, Sibille C, Dahan C, Mahfoudh W, BouchlakaSouissi C, et al. Contribution of the BRCA1 and BRCA2 mutations to breast cancer in Tunisia.J Hum Genet. 2007:52:915-920.

17. Abdulrashid K, AlHussaini N, Ahmed W. Thalib L. Prevalence of BRCA mutations among hereditary breast and/or ovarian cancer patients in Arab countries: systematic review and meta-analysis. BMC Cancer. 2019;19,256.

18. MansouriM, Derkaoui T, Bakkach J, Loudiyi A, Nourouti NG, Barakat A, et al. Screening of BRCA1 and BRCA2 germline mutations in unselected triplenegative breast cancer patients: A series from north of Morocco. Precis Med Sci. 2020;1-6.

19. Li H, Durbin R. Fast and accurate short read alignment with BurrowsWheeler transform. Bioinformatics. 2009;25:1754-60.
20. Matthijs G, Souche E, Alders M, Corveleyn A, Eck S, Feenstra I, et al. Guidelines for diagnostic next-generation sequencing. Eur J Hum Genet. 2016;24:1515.

21. McKenna A, Hanna M, Banks E, Sivachenko A, Cibulskis K, Kernytsky A, et al. The genome analysis toolkit: a MapReduce framework for analyzing nextgeneration DNA sequencing data. Genome Res. 2010;20:1297-303.

22. Richards S, Aziz N, Bale S, Bick D, Das S, Gastier-Foster J, et al. ACMG Laboratory Quality Assurance Committee. Standards and guidelines for the interpretation of sequence variants: a joint consensus recommendation of the American College of Medical Genetics and Genomics and the Association for Molecular Pathology. Genet Med. 2015;17:405-24.

23. Tazzite A, Jouhadi H, Nadifi S, Aretini P, Falaschi E, Collavoli A, et al. BRCA1 and BRCA2 germline mutations in Moroccan breast/ovarian cancer families: novel mutations and unclassified variants. GynecolOncol. 2012;3:687-692.

24. Uhrhammer N, Abdelouahab A, Lafarge L, Feillel V, Ben Dib A, Bignon Y-J. BRCA1 mutations in Algerian breast cancer patients: high frequency in young, sporadic cases. Int J Med Sci.2008;5:197-202.

25. Cherbal F, Bakour R, Adane S, Boualga K, Benais-Pont G, Maillet P. BRCA1 and BRCA2 germline mutations screening in Algerian breast/ ovarian cancer families. Dis Markers. 2010;28:377-384.

26. Mahfoudh W, Bouaouina N, Ahmed SB, Gabbouj S, Shan J, Mathew R, et al. Hereditary breast cancer in Middle Eastern and North African (MENA) populations: identifcation of novel, recurrent and founder BRCA1 mutations in the Tunisian population. MolBiol Rep. 2012;39:1037-46.

27. Laarabi FZ, Ratbi I, ChafaiElalaoui S, Mezzouar L, Doubaj Y, Bouguenouch L, et al. High frequency of the recurrent c.1310_1313delAAGA BRCA2 mutation in the North-East of Morocco and implication for hereditary breast-ovarian cancer prevention and control. BMC Research Notes.2017:10:188.

28. Dlamini Z, Francies FZ, Hull R, Marima R.Artificial intelligence (AI) and big data in cancer and precision oncology.ComputStructBiotechnol J. 2020;18:2300-11.

29. Lin $\mathrm{PH}$, Chen M, Tsai LW, Lo C, Yen TC, Huang TY, et al. Using next-generation sequencing to redefine BRCAness in triple-negative breast cancer. Cancer Sci. 2020;111:1375-84.

30. Turner NC, Telli ML, Rugo HS, Mailliez A, Ettl J, Grischke EM, et al. A Phase II Study of Talazoparib after Platinum or Cytotoxic Nonplatinum Regimens in Patients with Advanced Breast Cancer and Germline BRCA1/2 Mutations (ABRAZO). Clin Cancer Res. 2019;25:2717-24.

31. Litton JK, Rugo HS, Ettl J, Hurvitz SA, Gonçalves A, Lee KH, et al. Talazoparib in Patients with Advanced Breast Cancer and a Germline BRCA Mutation. N Engl J Med. 2018;379:753-763.

32. Garufi G, Palazzo A, Paris I, Orlandi A, Cassano A, Tortora G, et al. Neoadjuvant therapy for triple-negative breast cancer: potential predictive biomarkers of activity and efficacy of platinum chemotherapy, PARP- and immune-checkpoint-inhibitors. Expert OpinPharmacother. 2020;21:687-699.

33. Fitzpatrick A, Tutt A. Controversial issues in the neoadjuvant treatment of triple-negative breast cancer. TherAdv Med Oncol. 2019;11: 1758835919882581.

34. Stringer-ReasorEM, May JE, Olariu E, Caterinicchia V, Li Y, Chen D, et al. An open-label, pilot study of veliparib and lapatinib in patients with metastatic, triple-negative breast cancer. Breast Cancer Res. 2021;23:30.

35. Wong-Brown MW, Meldrum CJ, Carpenter JE, Clarke CL, Narod SA, Jakubowska A, et al. Prevalence of BRCA1 and BRCA2 germline mutations in patients with triple-negative breast cancer. Breast Cancer Res Treat. 2015;150:71-80.

36. Riahi A, Gourabi ME, Chabouni-Bouhamed H. Dissimilarity between sporadic, non-BRCA1/2 families and hereditary breast cancer, linked to BRCA genes, in the Tunisian population. Breast Cancer Tokyo Jpn. 2016;23:807-12

37. Mahfoudh W, Bettaieb I, Ghedira R, Snoussi K, Bouzid N, Klayech Z, et al. Contribution of BRCA1 5382insC mutation in triple negative breast cancer in Tunisia. J Transl Med. 2019;17:123.

38. Gaceb $\mathrm{H}$ Cherbal F Bakour ROuld-Rouis A, Mahfouf HY. Clinicopathological and Molecular Study of Triple-Negative Breast Cancer in Algerian Patients.PatholoOncolRes. 2018; 24:297-308.

39. Tun NM, Villani G, Ong K, Yoe L, Bo ZM. Risk of having BRCA1 mutation in high-risk women with triple-negative breast cancer: a meta-analysis. Clin Genet. 2014;85:43-48.

40. Armstrong N, Ryder S, Forbes C, Ross J, Quek RGW. A systematic review of the international prevalence of BRCA mutation in breast cancer. Clinical Epidemiology 2019; 11: 543-561.

41. Sharma P, Klemp JR, Kimler BF, et al. Germline BRCA mutation evaluation in a prospective triple-negative breast cancer registry: implications for hereditary breast and/or ovarian cancer syndrome testing. Breast Cancer Res Treat. 2014;145:707-714.

42. Robertson L, Hanson $\mathrm{H}$, Seal S, Warren-Perry M, Hughes D, Howell I, et al. and TNT Trial TMG, BCSC (UK). BRCA1 testing should be offered to individuals with triple-negative breast cancer diagnosed below 50 years. $\mathrm{Br} \mathrm{J}$ Cancer 2012;106:1234-38.

43. Lu K. Value of Family History in Common Cancers, Epidemiology: From Genetics to Practice Presentation, Health Services Research and Quality of Care Track. ASCO 2015.

44. Gadzicki D, Evans DG, Harris H, Julian-Reynier C, Nippert I, Schmidtke J, et al. Genetic testing for familial/hereditary breast cancer e comparison of guidelines and recommendations from the United Kingdom, France, the Netherlands and Germany. J Community Genet 2011;2:53e69. 
45. Hartman AR, Kaldate RR, Sailer LM, Painter L, Grier CE, Endsley RR, et al. Prevalence of BRCA mutations in an unselected population of triplenegative breast cancer. Cancer.2012;118:2787-95.

46. Gonzalez-AnguloAM, Timms KM, Liu S, Chen H, Litton JK, Potter J, et al. Incidence and outcome of BRCA mutations in unselected patients with triple receptor-negative breast cancer. Clin Cancer Res. 2011;17:1082-89.

47. Young SR, Pilarski RT, Donenberg T, Shapiro C, Hammond LS, Miller J, et al. The prevalence of BRCA1 mutations among young women with triplenegative breast cancer.BMC Cancer. 2009;9:86.

48. Muendlein A, Rohde BH, Gasser K, Haid A, Rauch S, Kinz E, et al. Evaluation of BRCA1/2 mutational status among German and Austrian women with triple-negative breast cancer. J Cancer Res ClinOncol.2015;141:2005-12.

49. Zhang, J. Walsh MF, Wu G, Edmonson M, Gruber TA, Easton J, et al. Germline Mutations in Predisposition Genes in Pediatric Cancer. N Engl J Med. 2015;373:2336-2346.

50. Ohmoto A, Yachida S, Morizane C. Genomic Features and Clinical Management of Patients with Hereditary Pancreatic Cancer Syndromes and Familial Pancreatic Cancer. Int J Mol Sci. 2019;20:561.

51. Norquist BM, Harrell MI, Brady MF, Walsh T, Lee MK, Gulsuner S, et al. Inherited mutations in women with ovarian carcinoma. JAMA Oncol. 2016;2:482-90.

52. Wong-Brown MW, Avery-Kiejda KA, Bowden NA, Scott RJ. Low prevalence of germline PALB2 mutations in Australian triple-negative breast cancer.Int J Cancer. 2014;134:301-305.

53. Zanti M, Loizidou MA, Michailidou K, Pirpa P, Machattou C, Marcou Y, et al.NGS Panel Testing of Triple-Negative Breast Cancer Patients in Cyprus: A Study of BRCA-Negative Cases. Cancers (Basel). 2020,12:E3140.

54. Dansonka-Mieszkowska A, Kluska A, Moes I, Dabrowska M, Nowakowska D, Niwinska A, et al. A novel germline PALB2 deletion in polish breast and ovarian cancer patients.BMC Med Genet.2010;11:20.

55. Heikkinen $\mathrm{T}$, Karkkainen $\mathrm{H}$, Aaltonen $\mathrm{K}$, Milne RL, Heikkila $\mathrm{P}$, Aittomaki $\mathrm{K}$, et al. The breast cancer susceptibility mutation PALB2 1592delT is associated with an aggressive tumor phenotype. Clin Cancer Res. 2009;15:3214-22.

56. Antoniou AC, Casadei S, Heikkinen T, et al. Breast-cancer risk in families with mutations in PALB2. N Engl J Med. 2014; 371: 497-506.

57. Cybulski C,Klu'zniak W, et al. Clinical outcomes in women with breast cancer and a PALB2 mutation: A prospective cohort analysis. Lancet Oncol. 2015, 16: 638-644.

58. De Brakeleer S, De Greve J, Desmedt C, Joris S, Sotiriou C, Piccart M. et al. Frequent incidence of BARD1-truncating mutations in germline DNA from triple-negative breast cancer patients.Clin Genet. 2016;89:336-340.

59. González-Rivera M, Lobo M, López-Tarruella S, Jerez Y, Del MonteMillán M, Massarrah T, et al. Frequency of germline DNA genetic findings in an unselected prospective cohort of triple-negative breast cancer patients participating in a platinum-based neoadjuvant chemotherapy trial. Breast Cancer Res Treat. 2016;156:507-515

60. Rofes P, Del Valle J, Torres-Esquius S, Feliubadaló L, Stradella A, Moreno-Cabrera JM, et al. BARD1 Pathogenic Variants are Associated with Triple-Negative Breast Cancer in a Spanish Hereditary Breast and Ovarian Cancer Cohort.Genes (Basel). 2021; 12:150

61. Suszynska M, Kluzniak W, Wokolorczyk D, Jakubowska A, Huzarski T, Gronwald J, et al. BARD1 is a Low/Moderate Breast Cancer Risk Gene: Evidence Based on an Association Study of the Central European p.Q564X Recurrent Mutation. Cancers (Basel).2019;11:740.

62. Castera L, Harter V, Muller E, Krieger S, Goardon N, Ricou A, et al. Landscape of pathogenic variations in a panel of 34 genes and cancer risk estimation from 5131 HBOC families. Genet Med. 2018; 20:1677-86.

63. Federici G, Soddu S. Variants of uncertain significance in the era of high-throughput genome sequencing: a lesson from breast and ovary cancers. J ExpClin Cancer Res. 2020;39:46.

64. Ricker C, Culver JO, Lowstuter K, Sturgeon D, Sturgeon JD, Chanock CR, et al. Increased yield of actionable mutations using multi-gene panels to assess hereditary cancer susceptibility in an ethnically diverse clinical cohort. Cancer Genet.2016;209:130-137.

65. Hoffman-Andrews L. The known unknown: the challenges of genetic variants of uncertain significance in clinical practice. J Law Biosci.2018;22:648-657.

66. National Comprehensive Cancer Network. Genetic/familial high-risk assessment: breast and ovarian. Version 1.2018, October 3, 2017.NCCN Clinical Practice Guidelines in Oncology (NCCN Guidelines)2017. Available from: https://www.nccn.org/. Accessed December 15,2017.

67. National Institute for Health and Care Excellence [homepage on the Internet]. Familial breast cancer: classification, care and managing breast cancer and related risks in people with a family history of breast cancer. NICE clinical guideline CG164; 2013. Available from:nice.org.uk/guidance/cg164. Accessed November 30, 2017.

68. London Cancer Alliance [Internet]. LCA breast cancer clinical guidelines; 2013 (updatedMarch 2016). Available from: http://rmpartners.cancervanguard. nhs.uk/wp-content/uploads/2017/03/lca-breast-cancerclinical-guidelinesoctober-2013-updated-march-2016-.pdf. Accessed November 30, 2017.

69. Forbes C, Fayter D, de Kock S, Quek RG. A systematic review of international guidelines and recommendations for the genetic screening, diagnosis, genetic counseling, and treatment of BRCA-mutated breast cancer.Cancer Manag Res. 2019;11:2321-37.
70. Gupta S, Provenzale D, Regenbogen SE, Hampel H, Slavin TP, Hall MJ, Llor X, et al. NCCN Guidelines Insights: Genetic/Familial High-Risk Assessment: Colorectal, Version 3.2017. J NatlComprCancNetw. 2017 Dec;15(12):1465-75.

71. Paluch-Shimon S, Cardoso F, Sessa C, Balmana J, Cardoso MJ, Gilbert F, et al. ESMO Guidelines Committee. Prevention and screening in BRCA mutation carriers and other breast/ovarian hereditary cancer syndromes: ESMO Clinical Practice Guidelines for cancer prevention and screening. Ann Oncol. 2016;27:v103-v110. Erratum in: Ann Oncol. 2017;28:iv167-iv168.

72. Li M, Yu X. Function of BRCA1 in the DNA damage response is mediated by ADP-ribosylation. Cancer Cell. 2013;23:693-704.

73. Zhu Y, Liu Y, Zhang C, Chu J, Wu Y, Li Y, et al. Tamoxifen-resistant breast cancer cells are resistant to DNA-damaging chemotherapy because of upregulated BARD1 and BRCA1. Nat Commun. 2018;9:1595.

74. Pinker, K., Chin, J., Melsaether, A.N., Morris, E.A., Moy, L. Precision Medicine and Radiogenomics in BreastCancer: New Approaches toward Diagnosis and Treatment.Radiology 2018, 287, 732-747.

75. Śniadecki M, Brzeziński M, Darecka K, Klasa-Mazurkiewicz D, Poniewierza P, Krzeszowiec M, et al. BARD1 and Breast Cancer: The Possibility of Creating Screening Tests and New Preventive and Therapeutic Pathways for Predisposed Women. Genes (Basel). 2020;11:1251.

76. Cavaillé $\mathrm{M}$, Uhrhammer $\mathrm{N}$, Privat $\mathrm{M}$, Ponelle-Chachuat $\mathrm{F}$, Gay-Bellile $\mathrm{M}$, Lepage $M$, et al. Feedback of extended panel sequencing in 1530 patients referred for suspicion of hereditary predisposition to adult cancers. Clin Genet. 2021;99:166-175. 